\title{
Desafíos de la inclusión escolar del niño con autismo
}

\author{
Challenges of school inclusion of the child with autism
}

\section{Sr. Editor:}

El espectro autista y los trastornos generalizados del desarrollo abarcan un amplio grupo de trastornos cognitivos y neuro-comportamentales que incluyen características que definen el núcleo de la socialización deteriorada, patrones de conducta restringidos y repetitivos y la alteración de la comunicación verbal y no verbal (1). Estas áreas implicadas representan limitaciones para los niños con trastorno del espectro autista (TEA) que, cuando no son intervenidas, dificultan su aprendizaje e inclusión en la escuela.

La inclusión educativa debe entenderse como una balanza equilibrada entre un aprendizaje y rendimiento escolar de calidad y congruente con las capacidades del estudiante que asegure un aprendizaje significativo para todos (2). En ese sentido, es relevante conocer los problemas que atraviesan los niños con TEA, así como las intervenciones en salud que abordan estas dificultades y mejoran su desempeño para la correcta fijación de aprendizajes en la escuela.

$\mathrm{Si}$ bien un aspecto que repercute significativamente en el progreso de los niños con TEA es el diagnóstico y tratamiento temprano, aún resulta tardío el reconocimiento de las características de estos niños fuera del ámbito clínico, situación que obstaculiza el aprovechamiento de las posibilidades de intervención que brinda la neuro plasticidad en los primeros años del desarrollo (3).

Las investigaciones realizadas han determinado que los niños y adultos con TEA conforman un grupo diverso, cuyas necesidades van a variar a lo largo de la vida en términos de evaluación como de intervención; en ese sentido, es necesaria una evaluación cuidadosa para determinar los servicios más apropiados de forma individualizada (4). Por tal razón la intervención temprana, atendiendo a las características individualizadas de cada niño con TEA y su familia, antes de que el niño asista al colegio es fundamental para desarrollar habilidades que faciliten su mejor desempeño en el ámbito escolar y permitan lograr la inclusión.

El autismo involucra varias áreas del desarrollo y es importante que un equipo multidisciplinario participe en su detección temprana e intervención, y de esa forma, favorecer el apoyo psicológico a los padres y la integración del niño a la escuela y a su medio (5). En el desarrollo de estrategias destinadas a facilitar el desempeño de los niños con TEA se requiere la participación temprana de diversos profesionales y el establecimiento de una cultura de diálogo continuo, constante y fluido entre ellos.

Instituto Nacional de Rehabilitación "Dra. Adriana Rebaza Flores” Amistad Perú - Japón. Lima, Perú.

Licenciada en Tecnología Médica Terapeuta Ocupacional 
Los padres de niños con TEA consideran que es insuficiente el personal educativo especializado en el conocimiento del autismo y que este grupo reducido aplica modelos jerárquicos y verticales, sin tomar en cuenta opiniones de personas que cuentan con experiencia directa en el conocimiento de estos niños, por ejemplo, los mismos padres de familia, auxiliares de clase u otro miembro no docente (6). Los padres o cuidadores no son expertos en todas las áreas que afectan al autismo; por ello, se requiere la orientación especializada de los profesionales involucrados. No obstante, es primordial durante el proceso de intervención, considerar los objetivos, necesidades y opiniones de las personas que conviven con estos niños para facilitar las interacciones entre los miembros de la familia.

La revisión de literatura realizada por Blanche y Reinoso (7), encontró el apoyo a la presencia de disfunciones sensoriales en el niño con TEA; estas disfunciones representan una alteración en la capacidad de respuesta (exacerbación o limitación). Muchas de esas disfunciones dificultan el desempeño de los niños con TEA dentro del aula, puesto que reciben una sobrecarga de información que obstaculiza su aprendizaje.

En los últimos años se ha incrementado los casos de niños con TEA en los que se solicitan terapias especializadas en integración sensorial con el objetivo de mejorar el procesamiento sensorial para construir respuestas adaptativas que conlleven a la organización del comportamiento; asimismo, en los niños que presentan dificultades de aprendizaje y problemas de procesamiento de integración sensorial, la colaboración entre el terapeuta y el equipo educativo surge como un enfoque efectivo, además del rol de los padres de coordinar los programas de salud y educación que funcionan bien para el niño (8). El trabajo conjunto entre los profesionales de salud, el personal educativo y los padres de familia coadyuvará la inclusión progresiva de los niños con TEA en el aula.

Por todo lo mencionado, la efectiva inclusión del niño con TEA en el ámbito escolar y el éxito de su desempeño, viene precedido de una concepción integral que implica la temprana detección e intervención y el abordaje ineludible del equipo multidisciplinario y los cuidadores. Hay mucho por esclarecer sobre el autismo; el trabajo colaborativo se perfila como la mejor opción para abordar la inclusión escolar de los niños con TEA.

\section{Giannina Valdez-Maguiña ${ }^{1, a}$, Rocio Cartolin- Príncipe ${ }^{1, a}$}

\section{Correspondencia:}

Giannina Valdez-Maguiña

Av. Prol. Defensores del Morro, cuadra 2, Distrito de Chorrillos 15057, Lima, Perú

Teléfono: 997352962

Correo electrónico: givamatmto@gmail.com

\section{REFERENCIAS BIBLIOGRÁFICAS}

1. Filipek PA, Accardo PJ, Ashwal S, et al. Practice parameter: Screening and diagnosis of autism. Neurology. 2000; 55(4):468-79.

2. Echeita G. Inclusión y exclusión educativa. «Voz y quebranto». REICE. 2008; 6(2):9-18.

3. Klin A, Klaiman C, Jones W. Rebajar la edad de diagnóstico del autismo: la neurociencia del desarrollo social afronta un importante problema de salud pública. Revista de Neurología. 2015; 60(Supl.1):S3-11.

4. Lord C, Bishop SL. Autism spectrum disorders: Diagnosis, prevalence, and services for children and families. Social Policy Report. 2010; 24(2): 3-21. (Fecha de acceso 22 de noviembre de 2018). Disponible en: https://eric.ed.gov/?id=ED509747

5. Cabezas H. Efecto de la aplicación de Terapia conductual en la adquisición de destrezas básicas para el aprendizaje, en tres niños con autismo. Revista Educación. 2004; 28(1):133-43.

6. Stanton M. Convivir con el autismo. Barcelona: PAIDÓs; 2002. p.1-133.

7. Blanche EI, Reinoso G. Revisión de la literatura: déficit de procesamiento sensorial en el espectro del autismo. Revista Chilena de Terapia Ocupacional. 2007; 7:59-68.

8. Ayres J. La integración sensorial en los niños. Madrid: TEA Ediciones; 2008.p.236.

Recibido: 10/12/2018 